\title{
Primary alveolar rhabdomyosarcoma of breast in a 13 year old female with cardiopulmonary metastases and cyto- histological correlation: A case report
}

\author{
Wamala $\mathrm{D}^{1, *}$, Kitayimbwa $\mathrm{P}^{1}$ and Dworak $\mathrm{O}^{2}$ \\ ${ }^{1}$ Department of Pathology, Mulago Hospital and Makerere University, Kampala, Uganda \\ ${ }^{2}$ Institute of Pathology, Furth Teaching Hospital of University of Erlangen, Furth, Germany
}

\begin{abstract}
Introduction: Rhabdomyosarcoma is the commonest soft tissue sarcoma of childhood. The tumour commonly occurs in the body regions of the head and neck, genitourinary and extremities. Primary rhabdomyosarcoma of the breast is extremely rare and present diagnostic challenges especially in resource limited centers. It is an aggressive tumour with a poor prognosis especially when diagnosed late. Case presentation: We present a case of 13 year old female with primary rhabdomyosarcoma of breast metastasized to regional axillary lymph nodes, lung and heart. The patient failed to respond to chemotherapy mainly due diagnostic challenges and succumbed to the disease. Conclusion: Breast masses in young patients should be diagnosed early and accurately, and optimal treatment promptly instituted. The incidence of primary rhabdomyosarcoma of breast is increasing in teenagers, and the tumour has a bad prognosis especially in late stage. We think this case will add knowledge and skills required in histological and cytological diagnosis of breast rhabdomyosarcoma.
\end{abstract}

Keywords: rhabdomyosarcoma; breast tumour; metastatic disease; cardiopulmonary metastases

\section{Introduction}

Rhabdomyosarcoma is the commonest soft tissue sarcoma of childhood, accounting for $5 \%$ of malignant tumors in this age category $[1,2]$. Two histological subtypes are seen in children, embryonal and alveolar. Alveolar rhabdomyosarcoma occur more commonly in adolescents while embryonal subtype is seen more often in younger children [3]. Common sites of primary disease include the head and neck region, genitourinary tract, and extremities [2]. Primary rhabdomyosarcoma of the breast is extremely rare and poses extreme diagnostic challenges especially in low resource countries where ancillary diagnostics tests are not available.

\section{Case presentation}

We present a case report of a juvenile primary breast alveolar rhabdomyosarcoma. A13 yearold Ugandanfemale presented with a right breast tumour which progressed from a small painless lump that developed soon after her menarche at $12 \mathrm{yrs}$. The patient had no other complaint by then. Her medical and family history was unremarkable. The patient had previously a right breast lumpectomy performed at a rural health facility. Observations made on the biopsy specimen were erroneously suggestive of fibroadenoma. The lump recurred in the same breast 3 months after surgery, this time associated with severe breast pain and right axillary lymphadenopathy clinically staged-T4N1Mx. The patient was referred to our institution one year after the right breast lumpectomy. She came with right breast tumour, associated nipple oedema and satellite areolar tumour nodules (Figure 1). Chest X ray revealed a right homogeneous opacity and a massive right pleural effusion. The pelvic and lumbar spinal X-rays were normal. Breast ultrasound report showed ill-defined heterogeneous solid mass with calcifications in the right breast covering areolar and sub areolar regions. There was another ill-defined echo complex mass in the lateral

*Corresponding author: Wamala D, Mulago Hospital and Makerere University, Kampala, Uganda, Email: dwamala@gmail.com

Received 9 May 2013 Revised 13 June 2013 Accepted 20 June 2013 Published 27 June 2013

Citation: Wamala D, Kitayimbwa P, Dworak O (2013) Primary alveolar rhabdomyosarcoma of breast in a 13 year old female with cardiopulmonary metastases and cyto-histological correlation: A case report. J Cancer Res Ther 1: 149-152. doi:10.14312/2052-4994.201323

Copyright: (C) 2013 Wamala D, et al. This is an open-access article distributed under the terms of the Creative Commons Attribution License, which permits unrestricted use, distribution and reproduction in any medium, provided the original author and source are credited. 
upper quadrant of the right breast measuring (27x16) mm. Doppler echocardiography revealed arrhythmias. Fine needle aspiration cytology of the right breast showed a highly cellular tumour composed ovoid to round small neoplastic cells with moderate abnormal mitotic figures (Figure 2).

A cytological diagnosis of malignant right breast neoplasm was made, differential of which included poorly differentiated carcinoma, rhabdomyosarcoma, Ewing's' sarcoma and lymphoma.

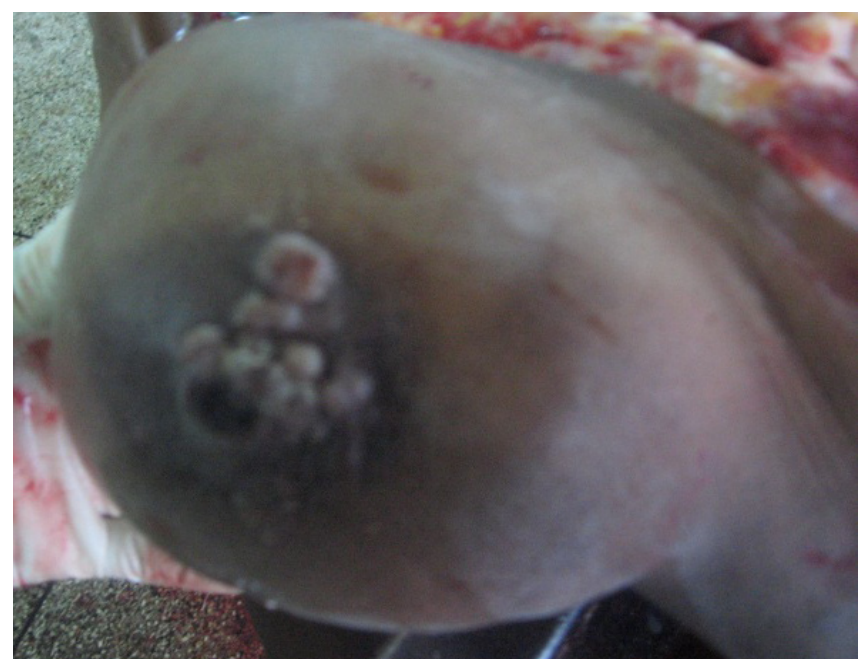

Figure 1 Primary breast rhabdomyosarcoma presenting as grossly swollen right breast with thickened nipple and areolar satellite nodules

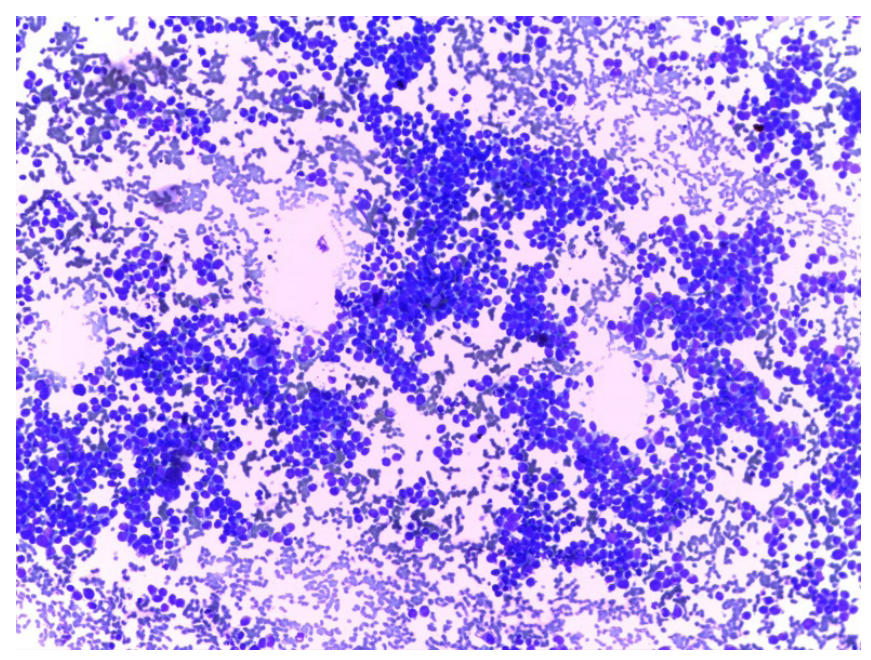

Figure 2 Cytomorphological features of breast alveolar rhabdomyosarcoma showing a highly cellular tumour composed ovoid to round small dyshesive neoplastic cells with scant cytoplasm, anisonucleosis together with numerous mitosis and apoptotic bodies

The patient was referred to the oncology unit where she completed 3 cycles of cyclophosphamide, adriamycin, $5 \mathrm{FU}$, granistenin and bleomycin for breast carcinoma with no improvement. A repeat surgical biopsy was performed. Histological findings included neoplastic cells with round to oval regular nuclei and a monotonous chromatin pattern. The cells formed aggregates interrupted by fibrovascular septae, and within these aggregates where areas of dyshesion suggestive of an alveolar rhabdomyosarcoma. Immunohistochemistry studies revealed that the neoplastic cells were negative for $\mathrm{AE} /$ AE3, LCA, S-100. Chromogranin, synaptophysin and TTF-1 but reactive for alpha- actin and desmin (Figures 3 and 4). A poorly differentiated or epitheloid leiomyosarcoma exhibit the same immunostaining profile but not the histomorphology of round small dark monotonous neoplastic cells separated by fibro vascular alveolar like septa seen in this case report. These findings and the histo-morphological pattern supported the diagnosis of Alveolar rhabdomyosarcoma, and excluded small cell carcinoma, lymphoma, Primitive neural ectodermal tumour (PNET) or epithelial neoplasm.

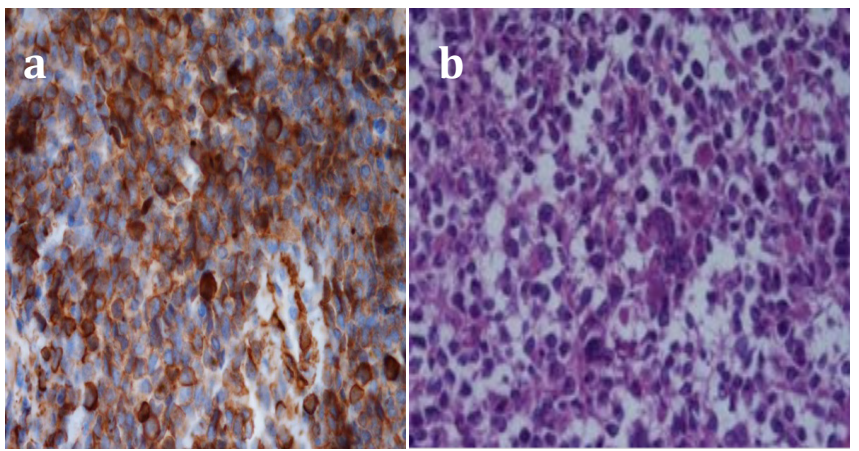

Figure 3 (a) Immunohistochemical study using actin revealed positive staining of tumour cells; (b) Haematoxylin \& Eosin stain section

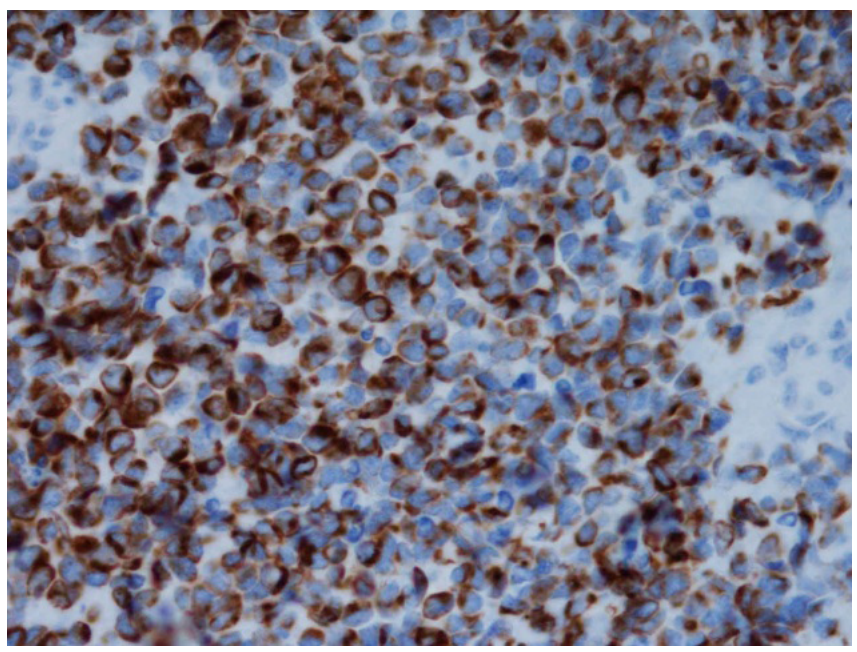

Figure 4 Immunohistochemical study using desmin revealed positive staining of tumour cells.

While in hospital, the patient developed respiratory distress with a high WBC count, and died. Autopsy examination revealed a Firm grey white tumor seeding predominantly the right pleura. The tumour was extensively infiltrating the pericardium and diaphragm both of which were markedly thickened. There were 200 $\mathrm{ml}$ straw colored right pleural effusion. Tumor involved the entire right lung, mainly infiltrating along the interstitium of the alveolar septae. A number of tumour nodules were seen on the heart (Figure 7) while both gross and histopathological findings were consistent with extensive tumour infiltration of the myocardium (Figure 5). 


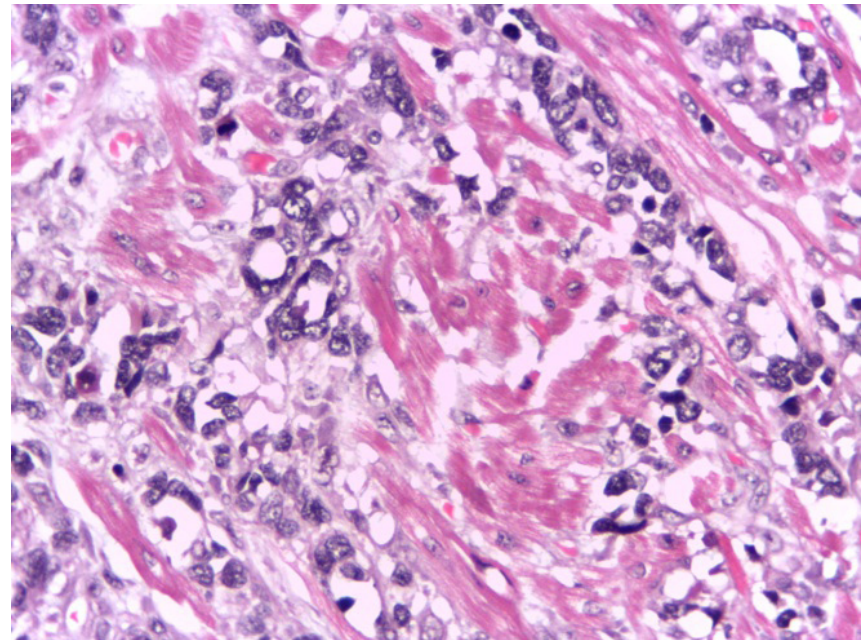

Figure 5 Tumour cells infiltrating the myocardium. X 100

\section{Discussion}

Rhabdomyosarcoma, malignant tumor of mesenchymal origin is the commonest childhood soft tissue tumour accounting for approximately $3.5 \%$ of the cases of cancer among children aged 0 to 14 years and $2 \%$ of the cases among adolescents and young adults aged 15 to 19 years [4]. The mostcommon primary sites of rhabdomyosarcoma are tumour the head and neck, genital urinary system and body extremities [2], it rarely presents as a primary tumour in the breast.

In childhood, there are three important histological types: the embryonal type (60\%) and the alveolar (20\%) [5]. The embryonal rhabdomyosarcoma predominantly seen in age group 0-4 commonly affects the genital urinary system and head and neck tends to have a better prognosis compared to the alveolar type which often develop in the extremities in children over 10 years, resistant to chemotherapy and metastasis early [6].

Primary rhabdomyosarcoma of the breast are extremely rare with previous studies done by the Intergroup Rhabdomyosarcoma Study group reporting only $7(0.2 \%)$ out of 3500 cases studied between 1972 and 1992 [7]. Most patients in young age subgroup 13.6 to 16.9 years (mean: 15.4 years), among the seven IRS patients subgroup studied six (86\%) had alveolar rhabdomyosarcoma while one (14\%) had embryonal rhabdomyosarcoma [7]. $50 \%$ of the patients presented with metastasis whereas three patients has previous diagnosis of fibroadenoma. Our patient had two previous erroneous diagnosis of fibroadenoma, the commonest breast tumour in this age group. This probably explains why diagnosis of rhabdomyosarcoma may be delayed. Mammography and ultrasound are utilized to locate the tumour but cannot distinguish it from a fibroadenoma. Hays et al. [7] advises fine needle aspiration cytology on teenage girls with breast tumour greater than $2 \mathrm{~cm}$.

The reactivity of immunohistochemical vimentin and desmin markers correlates with the degree of tumour cell differentiation, as it does in embryogenesis. Thus, only vimentin is present in the cytoplasm of the most primitive cells, and desmin and actin are acquired by developing rhabdomyoblasts. Antibodies against MyoD1 and myogenin are highly specific and sensitive for rhabdomyosarcoma and are currently used as standard antibodies for diagnosis [8]. We did not have MyoD1 and myogenin, so we depended on the characteristic morphological pattern of the tumour in addition to the available immunohistochemistry panel.

There are three major histological sub types of alveolar rhabdomyosarcoma: those with typical features, a solid pattern, and a mixed alveolar and embryonal appearance [3]. This case is an example of a typical alveolar rhabdomyosarcoma with round small dark monotonous neoplastic cells separated by fibro vascular alveolar like septa (Figure 6). Characteristic translocation $\mathrm{t}(2 ; 13)$ (q35;q14) between the long arm of chromosome 2 and the long arm of chromosome 13 and translocation $t(1 ; 13)$ (p36;q14)havebeenidentifiedinAlveolarRMS[9] resulting in PAX3-FKHR or PAX7-FKHR gene fusions respectively present in 18 of 21 alveolar rhabdomyosarcomas, while the embryonal rhabdomyosarcoma often exhibit deletion of the short arm of chromosome [10].

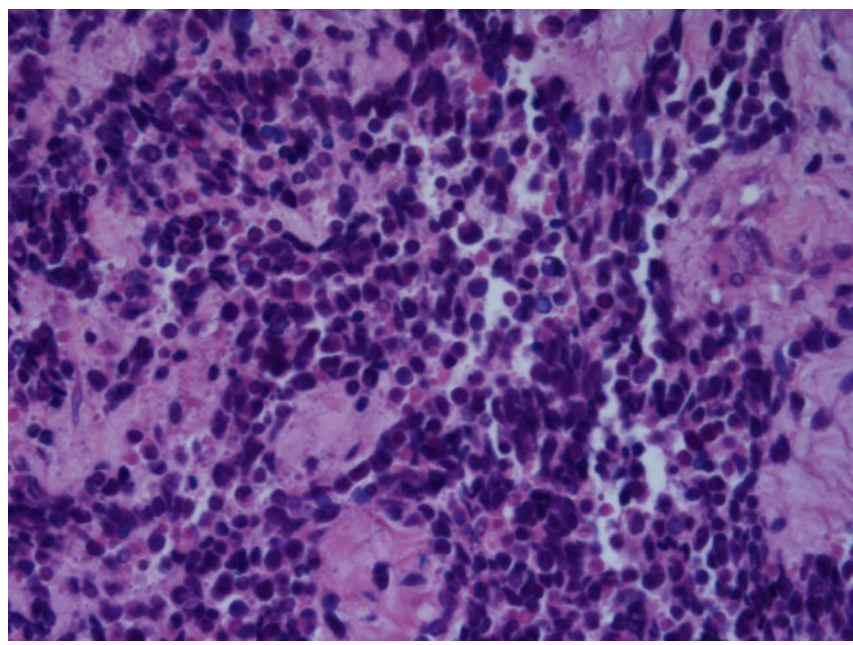

Figure $6 \mathrm{H}$ \& E section: Primary alveolar rhabdomyosarcoma of the breast, fibrovascular septa separate dark round monotonous neoplastic cells

Cytomorphological features of alveolar rhabdomyosarcoma consist of dyshesive round neoplastic cells with scant cytoplasm, anisonucleosis together with numerous mitosis and apoptotic bodies (Figure 4). Differentials include malignant small round cell tumour characterized by small dark round undifferentiated cells. These tumours includeEwing'sarcoma,synovialsarcoma,hepatoblastoma, granulocytic sarcoma, intrabdomonal desmoplastic small round cell tumour, small cell osteogenic sarcoma small cell carcinoma, Primitive Neural Ectodermal Tumour (PNET), Non Hodgkin's Lymphoma and Ewing's Sarcoma. These poorly differentiated tumours are difficult to diagnose basing on morphology alone ancillary test useful in diagnosis and classification are immunohistochemistry and flow cytometry immunophenotyping, fluorescence 
in situ hybridization, electron microscopy and reverse transcriptase polymerase chain reaction (RT-PCR) [11]. The alveolar rhabdomyosarcoma can be confirmed by a FISH utilizing a cytospin preparation to evaluate the neoplastic cells for the FKHR gene rearrangement on chromosome 13q14 [12]. Differentials can be excluded using immunocytochemical markers. Our panel included AE/AE3, LCA, S-100. Chromogranin, synaptophysin and TTF-1 Ewing's sarcoma tumour cells tends to be smaller and uniform and tend to have more finely granular and delicate chromatin pattern.

The prognosis of alveolar rhabdomyosarcoma is worse than that of embryonal rhabdomyosarcoma. Our patient presented with bad prognostic parameters namely the tumour site, patient age, histological type and distant metastases to the heart (Figure 7) and lungs (Figure 8).

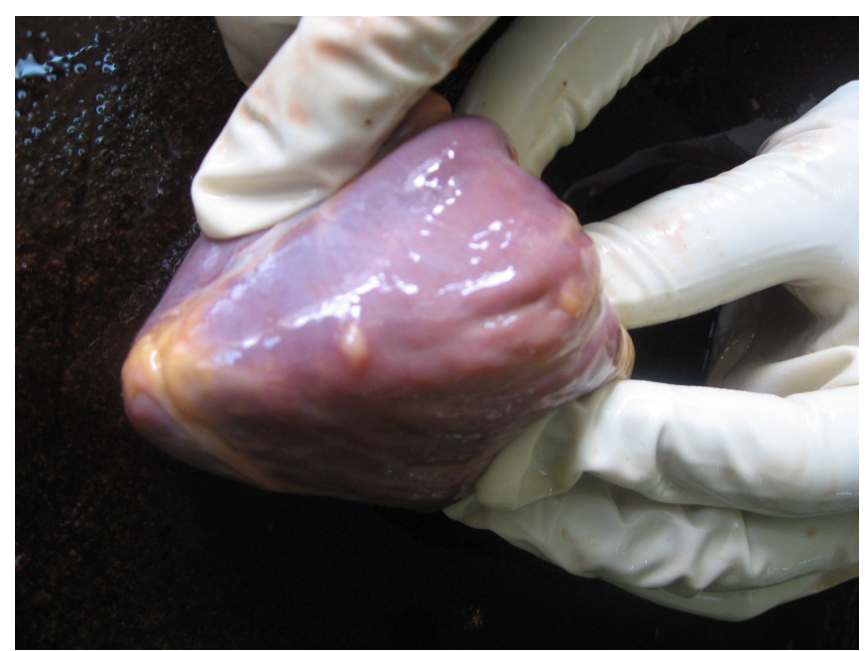

Figure 7 Secondary tumour nodules from primary breast alveolar rhabdomyosarcoma nodules on the heart.

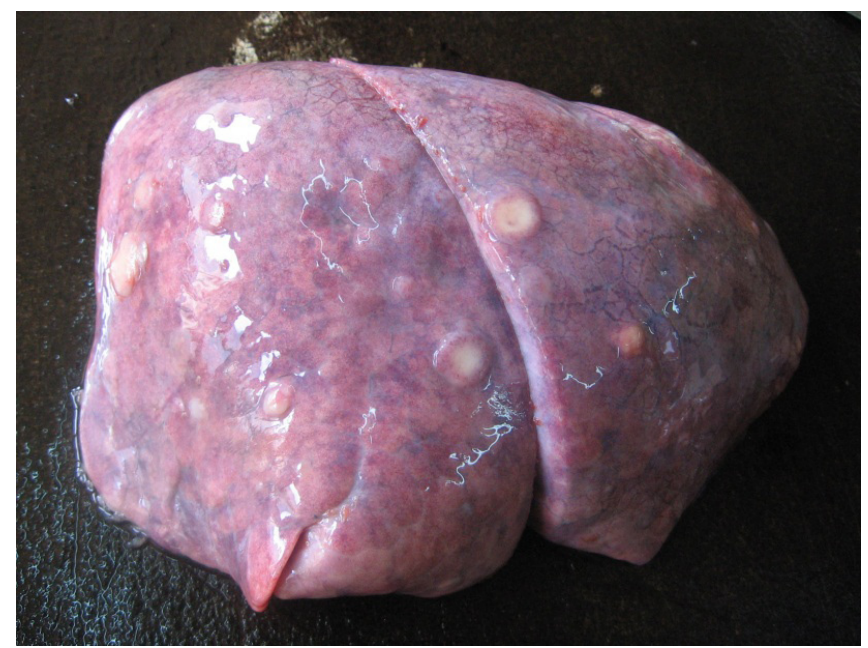

Figure 8 Secondary tumour nodules from primary breast alveolar rhabdomyosarcoma nodules on the lung

\section{Conclusions}

It is important for the medical team involved in management of young people with breast disease to ensure that accurate timely diagnosis and prompt optimal treatment of breast lumps and to be aware of the possibility of rhabdomyosarcoma, an increasingly common sarcoma in children. Fine needle aspiration is increasingly playing a pivotal role in good management of breast lumps especially in teenagers.

\section{Acknowledgements}

We are grateful to the patient's mother who kindly agreed and consented to the publication of this article. We appreciate the support of Institute of Pathology, Fuerth Hospital for all the immunohistochemistry tests that enabled the accurate diagnosis.

\section{Conflict of interest}

All the authors declare that they have no conflict of interest.

\section{References}

[1] Herrera LJ, Lugo-Vicente H (1998) Primary embryonal rhabdomyosarcoma of the breast in an adolescent female: a case report. J Pediatr Surg 33:1582-1584.

[2] Dagher R, Helman L (1999) Rhabdomyosarcoma: an overview. Oncologist 4:34-44.

[3] Legendre BW, Nelson BL (2008) Alveolar rhabdomyosarcoma of the paranasal sinuses. Head Neck Pathol 2:302-304.

[4] Gurney JG, Severson RK, Davis S, Robison LL (1995) Incidence of cancer in children in the United States. Sex-, race-, and 1-year agespecific rates by histologic type. Cancer 75:2186-2195.

[5] Wijnaendts LC, van der Linden JC, van Unnik AJ, Delemarre JF, Voute PA, et al. (1994) Histopathological classification of childhood rhabdomyosarcomas: relationship with clinical parameters and prognosis. Hum Pathol 25:900-907.

[6] Raney RB, Anderson JR, Barr FG, Donaldson SS, Pappo AS, et al. (2001) Rhabdomyosarcoma and undifferentiated sarcoma in the first two decades of life: a selective review of intergroup rhabdomyosarcoma study group experience and rationale for Intergroup Rhabdomyosarcoma Study V. J Pediatr Hematol Oncol 23:215-220.

[7] Hays DM, Donaldson SS, Shimada H, Crist WM, Newton WA Jr, et al. (1997) Primary and metastatic rhabdomyosarcoma in the breast: neoplasms of adolescent females, a report from the Intergroup Rhabdomyosarcoma Study. Med Pediatr Oncol 29:181-189.

[8] Cessna MH, Zhou H, Perkins SL, Tripp SR, Layfield L, et al. (2001) Are myogenin and myoD1 expression specific for rhabdomyosarcoma? A study of 150 cases, with emphasis on spindle cell mimics. Am J Surg Pathol 25:1150-1157.

[9] Turc-Carel C, Lizard-Nacol S, Justrabo E, Favrot M, Philip T, et al. (1986) Consistent chromosomal translocation in alveolar rhabdomyosarcoma. Cancer Genet Cytogenet 19:361-362.

[10] Barr FG, Chatten J, D'Cruz CM, Wilson AE, Nauta LE, et al. (1995) Molecular assays for chromosomal translocations in the diagnosis of pediatric soft tissue sarcomas. JAMA 273:553-557.

[11] Rajwanshi A, Srinivas R, Upasana G (2009) Malignant small round cell tumors. J Cytol 26:1-10.

[12] Das K, Mirani N, Hameed M, Pliner L, Aisner SC (2006) Fine-needle aspiration cytology of alveolar rhabdomyosarcoma utilizing ThinPrep liquid-based sample and cytospin preparations: a case confirmed by FKHR break apart rearrangement by FISH probe. Diagn Cytopathol 34:704-706. 